Final Performance Report for

\title{
THEORETICAL NUCLEAR PHYSICS AND ASTROPHYSICS
}

Name: Jianshi Wu, Professor of Physics

Institution: Fayetteville State University, NC

Date: March 11, 1998 DOE Contract: DE-FG05-94ER40883

This is a program which was proposed to start a research collaboration in Nuclear Physics and Astrophysics between Oak Ridge National Laboratory (ORNL) and Fayetteville State University, NC (FSU). This program supported a Principal Investigator from FSU, Dr. Jianshi $\mathrm{Wu}$, for one-month summer research each year and travel expenses from July 1, 1994 to June 30,1997 . The progress in research has been made in the following two directions:

a) periodic solutions of the time-dependent Hartree-Fock equations;

b) particle productions in heavy ion collisions.

It resulted in two publications:

"Periodic Numerical solutions of the time-dependent Hartree-Fock Equations", J.-S. Wu, K.C. Wong, M. R. Strayer amd M. Baranger, Phys.Rev. C 56, 857 (1997).

"Schwinger Particle-Production Mechanism for a Finite-Length Flux Tube with Transverse Confinement", Cheuk-Yin Wong, Ren-Chuan Wang and Jianshi-Shi Wu, Phys. Rev. D51, 3940 (1995).

and the renewed DOE research grant, DE-FG02-97ER41044 .

\section{PERIODIC SOLUTIONS OF THE TIME-DEPENDENT HARTREE-FOCK EQUATIONS}

\section{A. INTRODUCTION}

Functional integrals provide a fundamental framework to describe the microscopic behavior of a quantum system systematically and consistently. In the stationary-phase approxiDISTRIBUTION OF THS DOCUMENT is UMAMTED hn ${ }_{1}$ MASTER 


\section{DISCLAIMER}

This report was prepared as an account of work sponsored by an agency of the United States Government. Neither the United States Government nor any agency thereof, nor any of their employees, makes any warranty, express or implied, or assumes any legal liability or responsibility for the accuracy, completeness, or usefulness of any information, apparatus, product, or process disclosed, or represents that its use would not infringe privately owned rights. Reference herein to any specific commercial product, process, or service by trade name, trademark, manufacturer, or otherwise does not necessarily constitute or imply its endorsement, recommendation, or favoring by the United States Government or any agency thereof. The views and opinions of authors expressed herein do not necessarily state or reflect those of the United States Government or any agency thereof. 


\section{DISCLAIMER}

Portions of this document may be illegible electronic image products. Images are produced from the best available original document. 
mation (SPA) to an exact functional integral, the energy levels of collective states and fission decay widths can be obtained through the actions over periodic orbits of the time-dependent Hartree-Fock (TDHF) equations in real and imaginary time.

It has been known for some time that in the SPA bound state energies of a many-body system can be found from the periodic solutions of the time-dependent Hartree-Fock (TDHF) equations through a requantization rule [1]

$$
W_{I}=\int_{0}^{T} d t<\psi^{P}\left|i \partial_{t}\right| \psi^{P}>=2 m \pi
$$

where $\left|\psi^{P}\right\rangle$ is a periodic solution and $n$ is an integer. The stationary solution gives the ground state energy, and the periodic solutions lead to collective excitations.

However, numerical success in generating periodic solutions had not reached such a level that the requantization rules could be applied until a series of recent publications [3-5]. In the last a few years we developed a numerical algorithm to calculate periodic orbits of the TDHF equations. The crucial point for such success is to preserve conservation laws exactly (not approximately) in the time evolution through an appropriate discretization scheme. The algorithm we developed has been proved to be effective in bound state problems [4-5]. We have applied this approach to determine the monopole excitations for the spherical nuclei of ${ }^{4} \mathrm{He}$ and ${ }^{16} \mathrm{O}$. This research has resulted in a publication. We used the nucleus of ${ }^{4} \mathrm{He}$ as an example to demonstrate the algorithm which leads a converging sequence to a quasi-periodic solution. The applications to other nuclei, especially, the algorithm to determine nuclear collective states using periodic orbits, will be discussed in a series of future publications.

\section{B. The Key to Periodic Orbits}

As explained by the ref. [3], the crucial point in the algorithm of construct periodic orbits is to preserve the conservation laws in the time evolution exactly (not approximately) through an appropriate discretization scheme. It can be easily proved that the TDHF equation for an isolated system preserves the particle numbers and the total energy. However, 
most numerical schemes for time-dependent equations can only preserve the conservation laws approximately. Just an approximation is not good enough for a periodic orbit. The reason for the requirement on the exact conservation is as follows. Periodic solutions from the discretized equations if they exist appear as a family of infinite solutions on the same orbit for a certain energy (or a certain period). Any point on the orbit could be a starting point for a solution. The key for building the family of periodic solutions on the same orbit is that the number of independent equations must be less than the number of independent variables. The exact energy conservation plus the periodicity will play the role in reducing the number of equations by one. If the numerical scheme for discretization could preserve the energy conservation exactly, one of the equations in the set of equations for one-step time evolution can be replaced by the equation of energy conservation, i.e. $E_{n}=E_{n+1}, n=1,2, \ldots$. When we impose the periodic condition after $N$ time steps, the last time step gives an equation $E_{N}=E_{1}$. However, this equation can be derived from energy conservation for previous time steps since $E_{1}=E_{2}=\ldots=E_{N}$. Therefore, the number of independent equations is actually reduced by one. The exact energy conservation in the time evolution paves the way to the numerical success.

We will choose the reduced unit system where $\hbar=c=m=1$ throughout the discussion.

\section{The Numerical Algorithm for Exact Energy Conservation}

Consider the one-body TDHF equations

$$
i \frac{\partial}{\partial t} \psi_{\alpha}=-1 / 2 \nabla^{2} \psi_{\alpha}+w(\rho) \psi_{\alpha}
$$

where $w(\rho)$ is the one-body potential, assumed to be dependent only on the local density $\rho$. We write the time-discretized form of these equations as

$$
\left|\psi_{\alpha}^{n+1}\right\rangle-\left|\psi_{\alpha}^{n}\right\rangle=-i \epsilon H(n+1 / 2)\left(\left|\psi_{\alpha}^{n}\right\rangle+\left|\psi_{\alpha}^{n+1}\right\rangle\right) / 2
$$

where $n$ is the index for the $\mathrm{n}^{\text {th }}$ time step, whose size is $\epsilon$ and the indices for spacediscretization are suppressed. The one-body Hamiltonian $H$, assigned the time $n+1 / 2$, has 
to be determined. One condition on $H$ is that it be hermitian, which insures unitarity. This is obvious if one writes (3) as

$$
\left|\psi_{\alpha}^{n+1}\right\rangle=U(n+1 / 2)\left|\psi_{\alpha}^{n}\right\rangle
$$

with

$$
U(n+1 / 2)=\frac{1-\frac{1}{2} i \epsilon H\left(n+\frac{1}{2}\right)}{1+\frac{1}{2} i \epsilon H\left(n+\frac{1}{2}\right)} .
$$

Therefore the total particle number $\sum_{\alpha}\left\langle\psi_{\alpha}^{n} \mid \psi_{\alpha}^{n}\right\rangle$ is conserved.

To determine $H(n+1 / 2)$, we invoke the time- discretized version of energy conservation. The conserved energy $E$, which should be independent of $n$, is

$$
E=\sum_{\alpha}\left\langle\psi_{\alpha}^{n}\left|K+\mathcal{V}\left(\rho_{n}\right)\right| \psi_{\alpha}^{n}\right\rangle
$$

where $K$ is the kinetic energy operator.

According to (2), the one-body Hamiltonian must have the form

$$
H(n+1 / 2)=K+w(n+1 / 2) .
$$

Even though energy conservation is a global condition, it can be proved by adopting a simple local relation, namely

$$
w(n+1 / 2)=\frac{\rho_{n+1} \mathcal{V}\left(\rho_{n+1}\right)-\rho_{n} \mathcal{V}\left(\rho_{n}\right)}{\rho_{n+1}-\rho_{n}}
$$

that the total energy is conserved. Actually, it canbe proved that the energy conservation can be preserved exactly by any unitary transformation

$$
\left|\psi^{n+1}>=U(n+1 / 2)\right| \psi^{n}>
$$

for the time evolution, where $U(n+1 / 2)$ commutes with $H(n+1 / 2)$.

In a test of $10^{4}$-step run with double precision the total particle number can be exactly preserved and the total energy can be preserved from $10^{-12}$ up to $10^{-14}$. The periodicity of the orbits resulting from such a time evolution showed the stability of the iterative procedure. 


\section{The Periodic Orbits for ${ }^{4} H e$}

The solutions $\psi_{\alpha}$ that we seek for eq.(2) are quasi-periodic [3]. Truly periodic functions $\phi_{\alpha}$ can be defined by

$$
\psi_{\alpha}(\mathbf{r}, t)=e^{-i \lambda_{\alpha} t} \phi_{\alpha}(\mathbf{r}, t)
$$

with

$$
\phi_{\alpha}(\mathbf{r}, t+T)=\phi_{\alpha}(\mathbf{r}, t)
$$

The quantity $\lambda_{\alpha}$ is known as the chemical potential. The equation satisfied by $\phi_{\alpha}$ is

$$
i \frac{\partial}{\partial t} \phi_{\alpha}=\left(H-\lambda_{\alpha}\right) \phi_{\alpha}
$$

As a test to the algorithm, we consider the monopole vibrations of ${ }^{4} \mathrm{He}$, using a simple version of the Skyrme force, and we neglect the Coulomb interaction between protons. This definiteness leads to some simplifications in describing our algorithm since the remaining wave function is a one-dimensional s-wave radial equation with four-fold degeneracy.

In searching for the numerical solutions we first discretize this equation in space and time. With $n \epsilon$ denoting the time, the time evolution takes the form

$$
\left|u^{n+1}\right\rangle-\left|u^{n}\right\rangle=-i \epsilon[K+w(n+1 / 2)-\lambda]\left(\left|u^{n}\right\rangle+\left|u^{n+1}\right\rangle\right) / 2,
$$

where $n=1,2 \ldots N$ and the space index is again suppressed. The potential operator $w$ is diagonal in space and given by eq. (7). The iterative procedure we use to reach the periodic solution is quite general and can be summarized as follows.

- Initialization. We obtain an initial guess for the period $T_{0}$ and the parameter $\lambda_{0}$ from a trial wave function $\left|u_{0}^{1}\right\rangle$, which has a large component of an oscillating mode. We examine the trajectory of $\left\langle r^{2}\right\rangle$ in time with $\lambda=0$, and we determine an approximate period $T_{0} \simeq N \epsilon$. From the phase angle $\theta_{0}=\arg \left\langle u_{0}^{N+1} \mid u_{0}^{1}\right\rangle \simeq \lambda_{0} T_{0}$, we determine an initial value $\lambda_{0}$. 
- In the $\mathrm{k}^{\text {th }}$ iteration of the procedure, we follow the time evolution of the trial wave function $\left|u_{k}^{n}\right\rangle$ using eqs. (9) and (7). At the end of the time evolution we obtain a wave function $\left|u_{k}^{N+1}\right\rangle$. The perodicity condition should require $\left|u_{k}^{1}\right\rangle=\left|u_{k}^{N+1}\right\rangle$, but this condition is not satisfied until we have reached a periodic solution. At each step of the iterations we use $\theta_{k}=\arg \left\langle u_{k}^{N+1} \mid u_{k}^{1}\right\rangle=\Delta \lambda_{k} T_{k}$ to correct the value of $\lambda_{k}$ with

$$
\lambda_{k+1}=\lambda_{k}+\Delta \lambda_{k}
$$

For the intermediate steps, equations (9) and (7) are exactly satisfied.

- For a periodic solution there is one more equation to satisfy exactly,

$$
\left|u_{k}^{1}\right\rangle-\left|u_{k}^{N}\right\rangle=-i \epsilon\left(K+w_{k}(N+1 / 2)-\lambda_{k}\right)\left(\left|u_{k}^{1}\right\rangle+\left|u_{k}^{N}\right\rangle\right) / 2
$$

We use this equation to correct the trial wave function $\left|u_{k}^{1}\right\rangle$ and obtain a new wave function for the next iteration, $\left|u_{k+1}^{1}\right\rangle$. We first solve equation (10) for the wave function $\left|u_{k}^{1}\right\rangle^{\prime}$ from the given $\left|u_{k}^{N}\right\rangle$ and the potential $w_{k}(N+1 / 2)$, and then take a linear combination of $\left|u_{k}^{1}\right\rangle$ and $\left|u_{k}^{1}\right\rangle^{\prime}$ as the new trial wave function $\left|u_{k+1}^{1}\right\rangle$. Again we analyze $\left\langle r^{2}\right\rangle$ and obtain a new estimate for the period $T_{k+1}$.

We repeat the procedure given above until the desired precision for the periodic solution is reached.

Our calculations show that this procedure is stable, and the convergence can be reached consistently. The convergence of the periodicity is measured by the distance between $\left|u_{k}^{1}\right\rangle$ and $\mid u_{k}^{N+1}>$ defined as

$$
\sigma_{d}=\sqrt{\frac{1}{M} \sum_{j}\left|u_{k}^{1}(j)-u_{k}^{N+1}(j)\right|^{2}} .
$$

This value of $\sigma_{d}$ could reach the level of $10^{-12}$, while the laregest component of the wave function is about 0.1. Fig.1 shows the convergence sequence for $\sigma_{d}$ which was stopped at the desired precision level $\sigma_{d}<5 \times 10^{-9}$.

For heavier nuclei we may need more than one wave function to describe its collective states. We have extended the above formulation for spherical nuclei from ${ }^{4} \mathrm{He}$ to ${ }^{16} \mathrm{O}$ and 
${ }^{40} \mathrm{Ca}$. For the case of ${ }^{40} \mathrm{Ca}$ we had four wave functions including a second S-wave function at a higher energy. Except for the Gramm-Schmidt orthonormalization procedure for the second S-wave function used at the end of each iteration for the case of ${ }^{40} C a$, the above approach can be simply generalized to the system with multiple wave functions. The preliminary results also showed success for heavier nuclei like ${ }^{16} \mathrm{O}$ and ${ }^{40} \mathrm{Ca}$.

\section{E. Requantization}

Periodic orbits of the TDHF equation provide us an over-completed basis made up of time-dependent Hartree-Fock wave functions. This basis includes static Hartree-Fock solutions as special cases. At low energies its description coincides with the linear response theory or the random phase approximation (RPA). It can also describe large amplitude collective motion. However, it is still a classical description. Comparison with the experimentally observed spectra of nuclear collective excitations can only be made through requantization. The requantization rule, analogous to Bohr-Sommerfeld quantization rule, has been derived by many authors in different ways[1] and given by Eq. (1). A static Hartree-Fock solution corresponds to the case $m=0$.

The first periodic solution at certain period is most difficult since the approximate period is not known. It may take thousands of iterations to reach a periodic solution. Once the first solution is reached, we may use it as a trial solution for a neighboring period, and force the solution to change its energy. In this way we can move the solution from one energy to another until the the quantization condition is satisfied. For the nuclei of ${ }^{4} \mathrm{He}$ and ${ }^{16} \mathrm{O}$, we have obtained the quantized states at excitation energies $E_{H e}=26.491 \mathrm{MeV}$ and $E_{O}=29.493 \mathrm{MeV}[5]$.

\section{F. Future Development for This Approach}

There are several important directions for future development of this approach in nuclear physics. 
- Coulomb Force. The Skyrme force introduced in the last section represents the short range nature of the strong interaction. The future research includes the long range force in nuclei. This is essential, especially when we would like to extend this approach to spontaneous fission. Since the evolution operator contains the dependence on the wave function in the next time step, we have to solve the Coulomb Potential in each iteration for every time step. The introduction of a long range force into the problem will increase the computation immensely.

- Full Skyrme Force. The future research includes an extension of the algorithm to a full Skyrme Hamiltonian for heavy nuclei, where the numerical algorithm preserving the exact energy conservation is still a major issue. It is the momentum dependence of the Skyrme force which needs to be reconsidered since it introduces non-local interactions to the picture. Once the form for single-particle evolution operator $h_{\alpha}(n+1 / 2)$ is derived the iterative procedure will be straight forward. However, the non-diagonal matrix elements in $h_{\alpha}(n+1 / 2)$ will complicate its dependence on $\left|\phi_{\alpha}^{n+1}\right\rangle$ tremendously, especially when the momentum operator is a non-sparse matrix as seen in the B-spline collocation method.

- Higher Dimensional Problems. Spherical symmetry only works for spherical nuclei and monopole excitations. Monopole excitations mainly serve as a test ground for the algorithm. More interesting physics lies in giant resonances and quadrupole excitations. In these cases we have to reduce the space symmetry to a rotational symmetry and describe the nuclear system in two space dimensions. It is the goal of our proposed research to solve realistic problems in higher space dimensions.

In higher space dimensions, even though the algorithm may not change much, the increase in number of variables by a dimension factor (i.e. the number of mesh points in one-dimension) will cause a dramatic increase in computational resources. Therefore, we have to be very cautious in moving up in space dimensions, and will not do that until we could optimize the convergence of the algorithm in one dimension. 
- Important Physics To be Addressed.

A huge amount of nuclear data has been accumulated in nuclear multipole moments, and transition densities. Great success has been achieved in various models to decribe these data. Our reseach is an approache capable of calculating these quantities microscopically. The success of this approach may have tremendous impact on nuclear physics. The extension to multiple resonances has to be carried out in higher space dimensions. This is a challenge we have to face evantually.

\section{References}

[1] S.J. Levit, J.W. Negele, and Z. Paltiel, Phys. Rev. C21 1603 (1980); J.W. Negele, RMP 54, 913 (1982); and references therein.

[2] Ph. Chomaz, H. Flocard and D. Vautherin, Phys. Rev. Lett. 56, 1787 (1986).

[3] M. Baranger, AIP Conference Proceedings 347, 226 (1995).

[4] J.S. Wu, K.C. Wong, M.R. Strayer, and M. Baranger, Phys. Rev. C56, 857 (1997).

[5] J.S. Wu, K.C. Wong, M.R. Strayer, and M. Baranger, (to be published).

[6] J. Wu, J.J. Bai and R.Y. Cusson, Z. Phys. A335, 125 (1990).

\section{PARTICLE PRODUCTION IN HEAVY-ION COLLISIONS}

\section{A. Schwinger Mechanism for Particle-Production in a Finite Flux Tube}

The particle production mechanism studied by Schwinger [1] was originally used to calculate the probability for the production of particle-antiparticle pairs in a constant Abelian electric field in the whole infinite configuration space. A previous work [2] in this mechanism extended Schwinger's work to the case where the constant electric field exists only between two parallel condenser plates at a finite longitudinal separation but infinite in the transverse dimensions. Subsequently, this mechanism was further extended to an infinite flux tube with 
the confinement of a scalar field $m(r)$ in the transverse direction [3]. In this publication we investigate the case of a finite length flux tube with transverse confinement.

For the production of fermion-antifermion pairs in a flux tube we consider the motion of a fermion in a linear Abelian gauge field and a transverse confining scalar potential. We use a transformation to convert the Dirac equation for the fermion in cylindrical coordinates to a scalar equation, and the transmission amplitude can be obtained explicitly from the longitudinal equation by matching the wave function at the boundaries.

In the flux tube due to the confinement in the transverse direction the transverse mass assumes discrete values. Therefore, in evaluating the pair production rate the phase space integral in the transverse direction is replaced by a summation over the finite number of transverse states. For each transverse state the integral in the longitudinal momentum is restricted by the finite length of the flux tube. The finite size in the transverse direction will affect the rate of pair production a great deal at the production threshold.

\section{References}

[1] J. Schwinger, Phys. Rev. 82, 664 (1951).

[2] R.C. Wang and C.Y. Wong, Phys. Rev. D 38, 348 (1988).

[3] H.P. Paval and D. M. Brink, Z. Phys. C 51, 119 (1991).

\section{B. The Screening Effect of Pair Production in Ultra-Relativistic Nucleus-Atom Collisions}

The main goal of the Relativistic Heavy-Ion Collider (RHIC) currently under construction at Brookhaven National Laboratory is to create the quark gluon plasma phase of matter in central collisions. One of the promising signals of quark-gluon plasma phase transition is the hadronic production of lepton pairs. However,there are other fundamental processes resulting in lepton pair productions, in particular, the pair production in periphereral collisions, which produces the dominant background. Most of theoretical calculations are based 
on two-photon processes for pair production [1], or an approximation to the perturbation theory of QED to the second order. The validity of the second-order perturbation theory was experimentally tested at the CERN [2] in the beam-target experiments at $200 \mathrm{GeV} / \mathrm{amu}$. The results suggested that theory overestimated the pair production, especially, in the high momentum spectrum. One of the sources of the reduction was pointed out [3] by the cosmic ray group in Marshall Space Flight Center (MSFC), i.e. the screening effect in the target atom could reduce the production significantly.

In the collaboration with MSFC, we considered the screening effect due to the ThomasFermi distribution of the electron density, which results in an atomic form factor in terms of an incomplete $\Gamma$ function [4]. Since the nuclear form factor and the atomic form factor cover different ranges of momentum, factorization of the form factors provide us a good approximation. Preliminary calculations with the additional atomic form factor confirm that the reduction of the total cross section for the elctron-positron pairs is at $20 \%$ level for the experiments at CERN. The research is in progress. More calculations will reveal the reduction in the monetum distribution. The results could be compared with the CERN experimental data in detail. These results are very useful to the design of the pairmeter for cosmic ray experiment, where the collisions are indeed between cosmic ray nuclei and target atoms.

\section{References}

[1] J.-S. Wu, C. Bottcher, M.R. Strayer and A.K. Kerman, Ann. Phys. 210, 402 (1991) , and references therein.

[2] C.R. Vane, S. Datz, P.F. Ditter, H.F. Crause, C. Bottcher M.R. Strayer, and R.Schuch, H. Gao, and Hutton, Phys. Rev. Lett. 69, 1911 (1992).

[3] J.H. Derrickson, P.B. Eby, K.H. Moon, T.A. Parnell, D.T. King, J.C. Gregory, Y. Takahashi, and T. Ogata, Phys. Rev. A 51, 1253 (1995).

[4] J.-S. Wu, J. Derrickson, T.A. Parnell, and M.R. Strayer, (to be published). 